\title{
MoM - A Real Time Monitoring and Management Tool to Improve the Performance of Vehicular Delay Tolerant Networks
}

\author{
João A. F. F. Dias ${ }^{1}$, Joel J. P. C. Rodrigues ${ }^{1,2}$, Juan F. de Paz ${ }^{2}$, Juan M. Corchado ${ }^{2}$ \\ ${ }^{1}$ Instituto de Telecomunicações, Universidade da Beira Interior, Covilhã, Portugal \\ ${ }^{2}$ University of Salamanca, Spain \\ joao.dias@it.ubi.pt; joeljr@ieee.org; fcofds@usal.es; corchado@usal.es
}

\begin{abstract}
Vehicular Delay Tolerant Networks (VDTNs) appears as an innovative approach to deal with several issues faced by vehicular communications. By exploiting vehicles and deploying a DTN store-carry-and-forward paradigm with an Internet protocol over VDTN it is possible to enable asynchronous communications in the absence of end-to-end links. VDTNs also combine a bundle-oriented communication with an out-of-band signaling approach. To ensure that network nodes follow the protocol contributing to the welfare of the network, network monitoring and management functions play a key role. This paper proposes and describes a monitoring and management tool for VDTNs, called MoM, which collects realtime information from the network in order to improve the overall network performance. Conducted studies shown that MoM contributes to increase the bundle delivery probability and decreases the amount of wasted resources.
\end{abstract}

Keywords - Vehicular delay tolerant networks (VDTN); Network monitoring; Network management; Performance evaluation

\section{INTRODUCTION}

Lately, the search for Intelligent Transportation Systems (ITS) [1] that enable low-cost communications attracted significant attention from both scientific community and industry. From this demand, several vehicular networking approaches have been proposed enabling communications in a wide variety of scenarios. Such approaches also allow proposing novel applications and services. Networks for safety information dissemination, monitoring networks for data collection, and networks to share multimedia content are some examples. Vehicular networks may also be considered to provide connectivity to remote or rural regions where there is a lack of network infrastructure, which does not allow having an end-to-end connectivity. Although the advances already achieved by this kind of networks, there are still work to be done in order to deal with several challenges. For example, the high mobility of vehicles leads to frequent network topology changes and short contact durations, which can lead to several problems in achieving successfully data communications [2].

Trying to overcome some of the above-mentioned issues, some vehicular architectures have been proposed. Vehicular Ad Hoc Networks (VANETs) [3] exploit vehicles to enable data communications assuming that routing protocols establish end-to-end connectivity. This conjecture turns VANETs suitable to network partition, disconnection, and long delays. The store-carry-and-forward paradigm proposed by Delay Tolerant Networks (DTNs) [4] helps vehicular communications to solve some of the open issues found in VANETs. More recently and gathering some contributions from the previous architectures, such as the DTN store-carry-and-forward paradigm, Vehicular Delay Tolerant Networks (VDTNs) [5] were proposed with deployment of out-of-band signaling with two independent planes: control and data. VDTNs are also characterized to operate over the link layer allowing to aggregate large IP (Internet Protocol) datagrams sharing the same characteristics into bundles [6]. Bundles represent the VDTN protocol data unit. VDTNs also consider three different types of nodes: mobile, terminal, and relay. Mobile nodes (e.g., vehicles) travel between terminal nodes, which act as access points to the VDTN network and may perform as traffic sources and traffic sinks. Relay nodes are fixed devices with large storage capacity, usually placed at crossroads, aiming to improve the number of contact opportunities.

Although some improvements were already achieved by vehicular communications, there are still some technical challenges that must be overcome, such as the highly dynamic network topology, network disruption, node interactions, and limited network resources. Furthermore, limited network resources are one of the major problems faced by vehicular networks. Nodes with limited resources tend to diverge from the protocol in order to keep their data integrity. For example, they may deny contacts with other nodes to keep their data integrity, or in the other hand, if they accept a contact opportunity with other node, they may have to drop some messages (leading to messages loss) to have enough space to receive new ones. This situation has huge consequences not only in the overall network performance but also in the performance of other nodes.

To decrease the impact of such behavior in the network it is very important to afford vehicular networks with sophisticated tools that can rapidly and accurately detect network anomalies and constraints contributing to improve the overall network performance. This paper proposes a tool for VDTNs, called MoM, that operates in real time allowing to not only 
monitoring but also performing management actions. Thus, the paper reviews the related literature considering the most relevant contributions on monitoring and management approaches for delay-tolerant and vehicular communications, proposes an accurate tool capable to perform both monitoring and management functions in VDTNs, and executes studies to evaluate the performance of VDTNs considering the proposed tool using the most relevant routing protocols.

The remainder of this paper is organized as follows. Section II overviews several monitoring and management solutions proposed for delay tolerant and vehicular networks. Section III describes the main principles and characteristics of MoM, while Section IV focuses on its performance evaluation and demonstration. Finally, Section V concludes the paper pointing some future work.

\section{RELATED WORK}

This section overviews the most relevant contributions related to network monitoring and management approaches proposed for delay-tolerant and vehicular communications, which may contribute to the development of MoM.

Wang et. al [7] proposed a compressive sensing based approach, called CSM, for vehicular networks monitoring. It aims to achieve high estimation accuracy using vehicles measurements. To collect vehicles information, CSM sets a few number of vehicles as seeds. This kind of vehicle is responsible to collect data from regular vehicles and deliver it to network access points (APs). APs consider k-NN estimate and Gaussian process regression to process vehicles measurements.

In the study presented in [8], the authors proposed a framework that is capable to optimize the process of traffic monitoring. This optimization is achieved by two delaytolerant routing protocols that collect vehicle information about traffic and global traffic statistics to accomplish routing forwarding strategy decisions that helps to minimize communication costs.

A monitoring and control system for DTNs is proposed in [9]. This system works in a distributed way allowing collecting the operational status of DTN nodes in order to detect operation failures and helps to seek solutions to solve these operational failures. Moreover, it also affords DTNs with a tool for diagnose and treatment failures where network operators may compose several failure treatment methods according to the collected reports. A set of monitoring mechanisms are presented in [10] to enable monitoring capabilities in the bundle and lower layers of the DTN architecture. These mechanisms focus on several important features, such as nodes available storage capacity, since the lack of storage capacity may lead to bundles loss. To gather network statistics the authors implemented ping bundles, which forces nodes to generate reports to be delivered to the monitor processor.

Nobre et. al [11] proposed a self-handling service to perform management functions in DTNs considering peer-topeer technologies. The service exploits the DTN concept to support and grouping management messages, and it can be used by system administrators for monitoring and heal managed network elements.
In [12], the authors proposed several policies to deal with buffer management in DTNs using global knowledge about the network. With a distributed algorithm that learns from the collected statistical data, these policies managed to increase the average delivery rate and drop the average delivery delay. Another buffer management policy is proposed in [13]. This policy has the capability to prioritize messages in nodes buffer allowing maximizing the successful delivery rate in the entire network. This is accomplished considering a generic replication-based routing model for message multicasting. A similar approach is followed by the work proposed in [14].

Regarding VDTNs, several proposals for monitoring and management purposes were already proposed. They take into consideration MMAN [15], which uses mobile nodes to perform monitoring functions and a set of monitoring stations to create an accurate update state of the network. An example of this is an application-layer approach [16] proposed to collect load-related information from VDTN nodes considering the Simple Network Management Protocol (SNMP). In [17], a management system to perform management functions using SNMP is proposed. Contrary to both approaches that uses SNMP to only perform monitoring or management functions, the proposal of MoM takes advantages of a backbone system and the VDTN out-of-band signaling to perform both monitoring and management functions.

\section{MOM SYSTEM}

This section describes the main characteristics and functionalities of MoM tool proposed for VDTNs. Its main goal is to afford VDTNs with an accurate tool capable to not only perform monitoring functions but also to take measures, in real time, when some network constraint is detected.

\section{A. Main Concepts}

As above-presented, the VDTN architecture deploys an out-of-band signaling with control and data plane separation. At the control plane nodes exchange signaling messages containing crucial information about their state (e.g., buffer capacity, power, location, and speed) that can be used not only to reserve resources to the data plane but also to evaluate their performance in the network.

The MoM tool takes advantages of this architectural approach to collect statistical information about the performance of each network node and to spread across the network measures to held constraint nodes or to take actions against nodes responsible for decreasing the network performance. To perform both monitoring and management functions, MoM requests nodes to collect reports about three kinds of statistics: node state, bundles traffic, and link statistics. Node state information is related to nodes available buffer space, speed, and location. Bundle traffic information is related to how many bundles each node receives, transmits, delivers, and drops, while link statistics allows MoM to know the amount of data transmitted and the amount of time a node spend to communicate with others.

An example of MoM operation can be described as follows. Each mobile node during its tour along the network keeps record of the number of delivered, sent, received, aborted, and dropped bundles. It also collects the volume of bytes 
exchanged and the amount of time spent in contact with other nodes. When a contact opportunity with a terminal node is available, it uses the control plane to send its statistical report. Then, terminal nodes send the received data to MoM to be processed. MoM processes the received information considering three different modules: monitor module, management module, and interface module. In case of network constraint detection, an advertisement is generated and spread across the VDTN network. Figure 1 illustrates the interactions between network nodes and MoM.

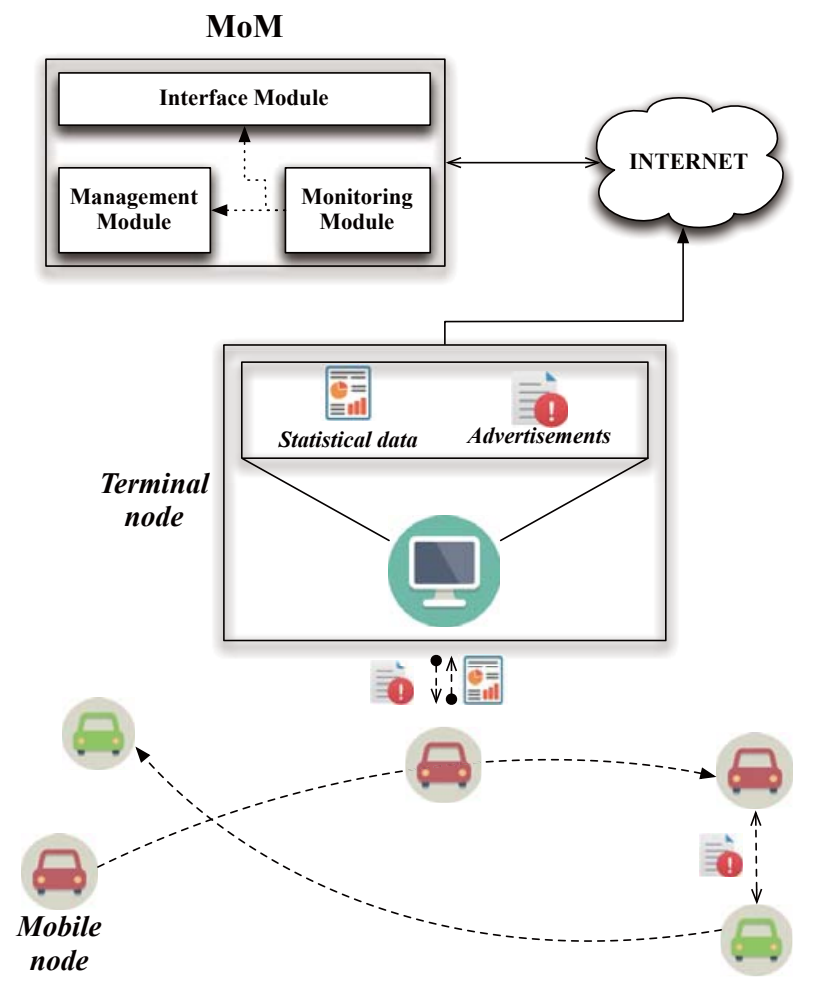

Fig. 1. Illustration of the interactions and exchanged data between MoM and VDTN network nodes.

\section{B. Monitoring Module}

The monitoring module is responsible to process mobile nodes reports and to keep updated a network state table containing all the relevant information about each network node. After processing the received data, it updates the state table and sends the updated information to the interface module in order to be presented to network administrators. At the same time, the monitor module evaluates each network node behavior assigning a performance coefficient $(P)$. This coefficient is calculated according to Equation 1, where $S$ is the number of sent bundles, $D$ the number of bundles delivered to the final destination, $C$ the amount of time spent communicating with other nodes, and $V$ the total volume of bytes exchanged with others. All the considered values are normalized to an interval between $[0,1]$ considering (2). After calculating all the network nodes performance coefficient, it calculates the performance coefficient for the entire network $\left(N_{p}\right)$ that is determined by Equation 3. Each time the monitoring module detects that a node has a $P$ below $N_{p}$ it notifies the management module.

$$
\begin{gathered}
P_{n}=\alpha \cdot S_{n}+\beta \cdot D_{n}+\gamma \cdot V_{n}+\delta . C_{n} \\
\text { where } \alpha, \beta, \gamma \text { and } \delta=\text { constant values } \\
\qquad X_{\text {normalized }}=\frac{x-\min (x)}{\max (x)-\min (x)} \\
N_{p}=\frac{\sum_{i=1}^{N} P_{i}}{N}
\end{gathered}
$$

Another constraint the monitoring module is always searching is for nodes closer to reach their full buffer capacity. This situation can contribute to the network performance deterioration since nodes with low buffer space may be forced to drop bundles to continuing receiving bundles from other nodes. This can lead to drop bundles that do not have any copy in the network comprising their changes to reach the final destination. To overcome this situation, each time a node with low buffer capacity is detected, the management module is informed.

\section{Management Module}

This module is responsible to generate network advertisements when it is notified by the monitor module. These advertisements are generated aiming to inform nodes about a behavior they must adopt or an action they must have against others. For example, the management module may generate an advertisement to alert nodes that a node is closer to reach its full buffer capacity. Receiving this kind of advertisement may lead nodes to avoid contacts with constraint nodes in order to not only save networks resources but also to prevent bundles to be dropped without reaching their final destination. Another example of advertisement that is generated by this module is an advertisement requesting a specific node to spend more time sending bundles from others or to use a higher percentage of its buffer to store bundles from others. This approach aims to increase the performance of nodes where $P$ is below $N_{p}$. All advertisements generated by the management module are sent to terminal nodes in order to being spread across the network.

\section{User Interface Module}

This module is used to display to network administrators the state of the monitored VDTN. The information sent by the monitoring module is processed into two main categories: network performance and nodes state. For the network performance, the processed information is used to show realtime network performance graphs about bundles (e.g., created, delivered, delivered delay, sent, received, aborted, and dropped), contact time, and exchanged bytes. The network tab of MoM interface module is shown in Figure 2. The node state tab displays several characteristics about each network node, such as buffer total capacity, free buffer space, speed, and location. It is also possible to know detailed information about which bundles nodes were carrying last time they enter into contact with a terminal node. Figure 3 shows the nodes tab of the MoM interface module. 


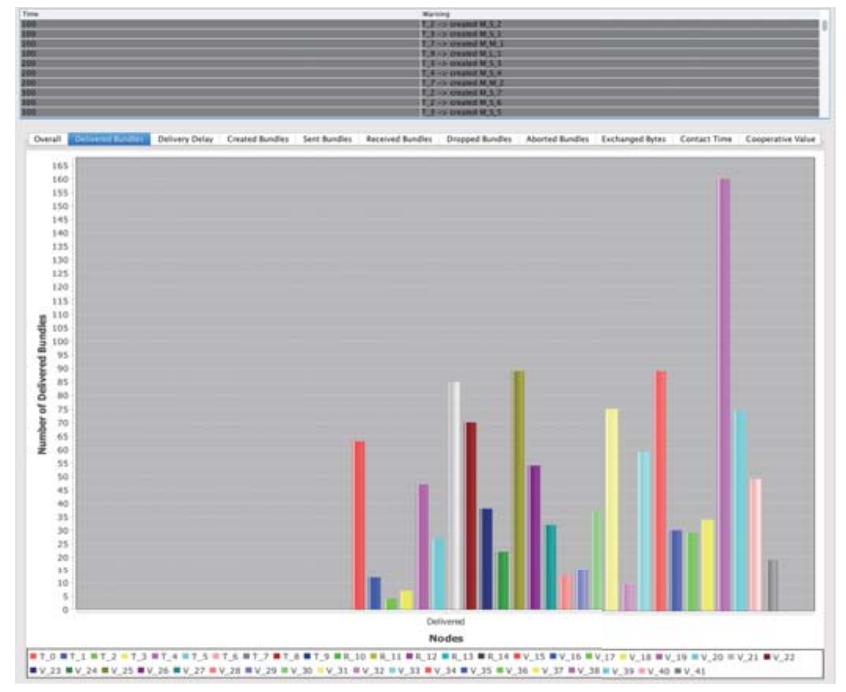

Fig. 2. Network tab of the MoM interface module showing a realtime graph about the number of delivered bundles by each mobile node.

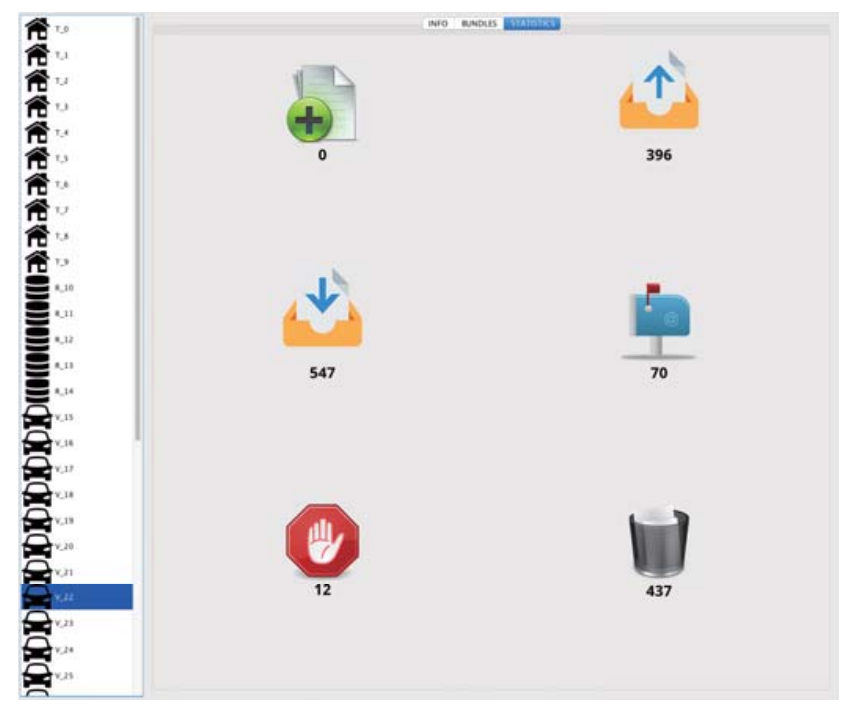

Fig. 3. Nodes tab of the MoM user interface module showing the number of created, sent, received, delivered, aborted, and dropped bundles for a specific node.

\section{MOM System EVALUATION AND DEMONSTRATION}

This section presents a case study to evaluate the performance of the MoM system and show the benefits it introduced in the VDTN architecture. The performance evaluation of MoM system was conducted through the VDTNSim [18] simulation tool.

\section{A. Simulation Scenario}

The simulation scenario considers a representation of a small part of the city of Helsinki, Finland (Fig. 4). During a simulation time of $24 \mathrm{~h}$, VDTN nodes establish communications among them considering a transmission range of 350 meters enabled by IEEE $802.11 \mathrm{~b}$ (at $6 \mathrm{Mbps}$ ) omnidirectional antennas. Ten terminal nodes with $100 \mathrm{MB}$ of buffer capacity generate bundles with sizes uniformly distributed in the range of $[50 \mathrm{~KB}, 750 \mathrm{~KB}]$ assuming a creation interval in a range of $[30,120]$ seconds. Five relay nodes with $200 \mathrm{MB}$ of buffer capacity are placed according to the map positions illustrated in Figure 4. To create different evaluation scenarios to better evaluate the impact of MoM on VDTNs, the number of mobile nodes with $50 \mathrm{MB}$ of buffer capacity changed between $25,50,75$, and 100 . In all the scenarios, the configuration of MoM parameters is set according to Table I. Performance metrics considered in all the experiments are the number of delivered bundles, the number of dropped bundles, and the protocol overhead ratio.

\begin{tabular}{ccccc}
\multicolumn{4}{c}{ TABLE I. } & \multicolumn{4}{c}{ MOM PARAMETERS } \\
\hline \hline Node Type & $\boldsymbol{\alpha}$ & $\boldsymbol{\beta}$ & $\boldsymbol{\gamma}$ & $\boldsymbol{\delta}$ \\
\hline \hline $\begin{array}{c}\text { Terminal } \\
\text { and Relay } \\
\text { Mobile }\end{array}$ & 0.7 & 0.0 & 0.15 & 0.15 \\
\hline \hline
\end{tabular}

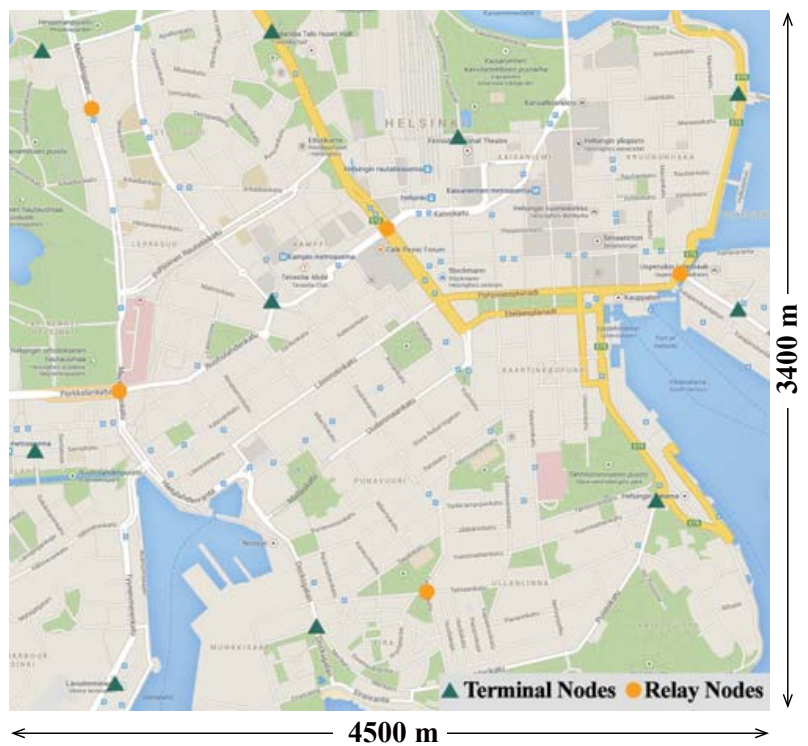

Fig. 4. Illustration of the map used to evaluate the performance of MoM, with the position of terminal and the stationary relay nodes.

\section{B. Perfomance Analysis}

The results obtained in the conducted experiments shown the importance of afford the VDTN architecture with a tool capable to handle some kinds of network constraints or diverging behaviors from network nodes. Figure 5 shows that followed approach by MoM system improves the overall network performance since more bundles are delivered to their final destination. This is achieved by constantly monitoring the network in searching nodes that are performing lower than the rest and requesting them to improve their contributions to achieve a better overall network performance. For instance, with the MoM tool activated the VDTN network achieves to 
delivered more 56, 265, 157, and 236 bundles (for a number of mobile nodes equals to $25,50,75$, and 100 , respectively) when compared to a scenario without MoM.

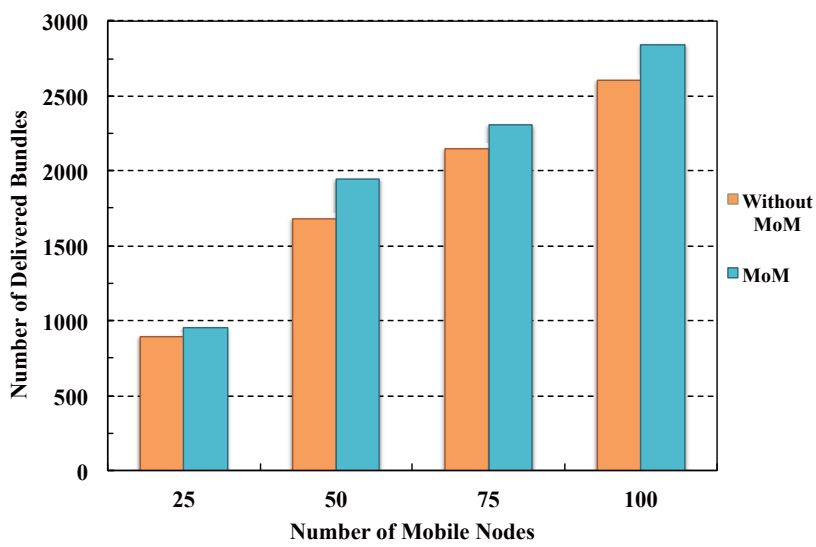

Fig. 5. Number of delivered bundles as function of the number of mobile nodes.

MoM contributions are not limited to the increase of the number of delivered bundles. It also contributes to reduce the amount of wasted resources. By detecting nodes closer to achieve their buffer capacity and spreading advertisements to the network, it allows other nodes to avoid contacts with constraint nodes. This approach allows nodes to avoid dropping unnecessary bundles to receive bundles from others. This may also contributes to increase the number of delivered bundles since it can be prevented that bundles are dropped at their final hop or without having any copy of them replicated across the network. Figure 6 confirms the above-presented conclusions. With MoM activated, VDTN network drops less 515, 781, 693, and 648 bundles. Consequently, this approach also contributes to decrease the protocol overhead ratio (Figure 7), which represents the number of bundle copies needed to deliver it to its final destination. These results demonstrated that MoM is also efficient not only in terms of storage but also in terms of bandwidth resources utilization.

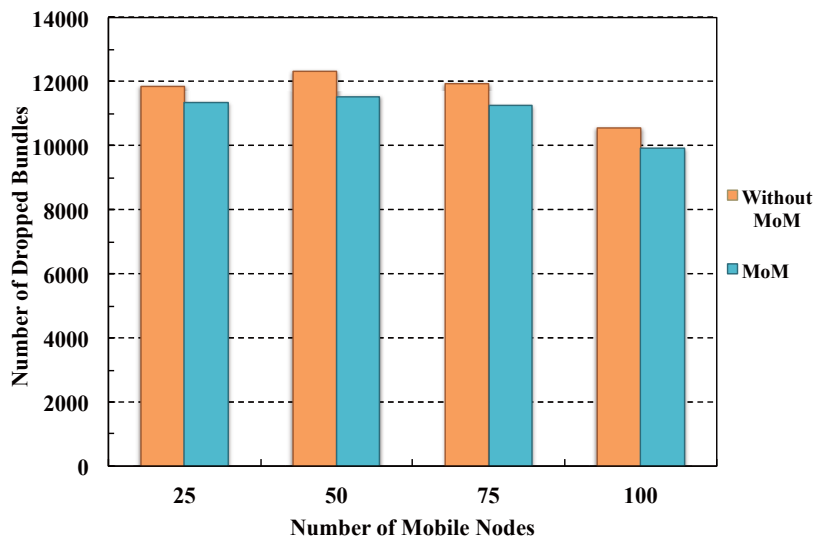

Fig. 6. Number of dropped bundles as function of the number of mobile nodes.

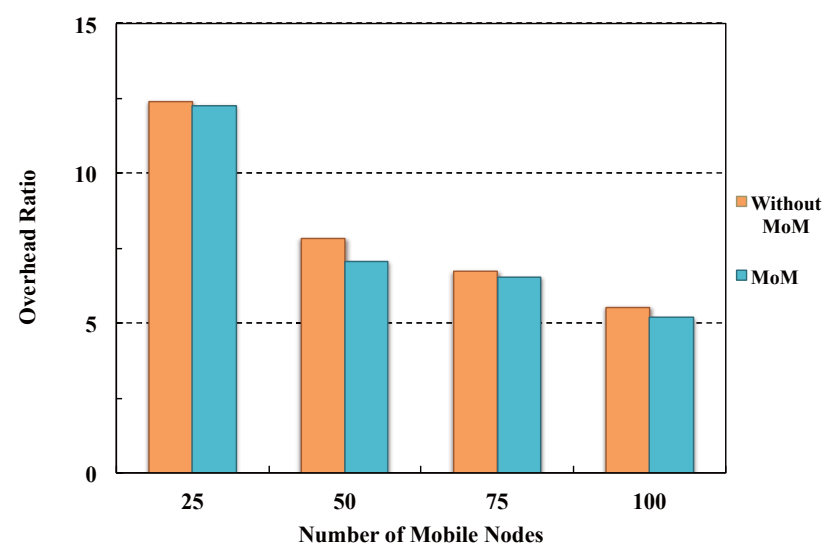

Fig. 7. Protocol overhead ratio as function of the number of mobile nodes.

\section{CONCLUSION AND FUTURE WORK}

This paper presented and evaluated the network performance of a real-time monitoring and management tool for VDTNs, called MoM. This robust and accurate tool constantly monitors the network searching for nodes with resource limitations or with a behavior that may comprise the overall network performance. When it finds an occurrence it generates advertisements with some guidelines that nodes should follow. These advertisements are spread across the network taking advantages of the VDTN out-of-band signaling at the control plane phase.

Performance studies showed that MoM tool benefits to achieve a better network performance when compared to an approach where nothing is considered. With MoM the number of delivered bundles increases and the amount of resources wasted decreases. Future work may focus on the deployment and performance evaluation of MoM in a laboratory VDTN testbed [19].

\section{ACKNOWLEDGMENTS}

This work has been partially supported by the Instituto de Telecomunicações, Next Generation Networks and Applications Group (NetGNA), Portugal, by National Funding from the FCT - Fundação para a Ciência e a Tecnologia through the UID/EEA/500008/2013 Project and the research grant SFRH/BD/86444/2012.

\section{REFERENCES}

[1] Z. Junping, W. Fei-Yue, W. Kunfeng, L. Wei-Hua, X. Xin, and C. Cheng, "Data-Driven Intelligent Transportation Systems: A Survey," IEEE Transactions on Intelligent Transportation Systems, vol. 12, Issue 4, pp. 1624-1639, 2011.

[2] G. Karagiannis, O. Altintas, E. Ekici, G. Heijenk, B. Jarupan, K. Lin, and T. Weil, "Vehicular Networking: A Survey and Tutorial on Requirements, Architectures, Challenges, Standards and Solutions," IEEE Communications Surveys \& Tutorials, vol. 13, Issue 4, pp. 584-616, 2011.

[3] A. Kumar and M. Sinha, "Overview on vehicular ad hoc network and its security issues," in 2014 International Conference on Computing for Sustainable Global Development (INDIACom), 5-7 March 2014, 2014, pp. 792-797. 
[4] S. M. Tornell, C. T. Calafate, J. C. Cano, and P. Manzoni, "DTN Protocols for Vehicular Networks: An Application Oriented Overview," IEEE Communications Surveys \& Tutorials, vol. 17, Issue 2, pp. 868-887, 2015.

[5] J. N. G. Isento, J. J. P. C. Rodrigues, J. A. F. F. Dias, M. C. G. Paula, and A. Vinel, "Vehicular Delay-Tolerant Networks - A Novel Solution for Vehicular Communications," IEEE Intelligent Transportation Systems Magazine, vol. 5, Issue 4, pp. 10-19, 2013

[6] V. N. G. J. Soares, F. Farahmand, and J. J. P. C. Rodrigues, "A layered architecture for Vehicular Delay-Tolerant Networks," in IEEE Symposium on Computers and Communications 2009 (ISCC 2009), 5-8 July 2009, 2009, pp. 122-127.

[7] W. Hongjian, Z. Yanmin, and Z. Qian, "Compressive sensing based monitoring with vehicular networks," in Proceedings of 2013 IEEE International Conference on Computer Communications (INFOCOM 2013), Turin, Italy, 14-19 April, 2013, pp. 2823-2831.

[8] A. Skordylis and N. Trigoni, "Efficient Data Propagation in Traffic-Monitoring Vehicular Networks," IEEE Transaction on Intelligent Transportation Systems, vol. 12, Issue 3, pp. 680-694, 2011.

[9] W. Shin-Ywan, "Distributed interplanetary Delay/Disruption Tolerant Network (DTN) Monitor and Control system," in IEEE Aerospace Conference, Big Sky, MT, USA, 3-10 March, 2012, pp. $1-9$.

[10] A. Papalambrou, A. G. Voyiatzis, D. N. Serpanos, and P. Soufrilas, "Monitoring of a DTN2 network," in Baltic Congress on Future Internet Communications (BCFIC 2011), Riga, Latvia, 16-18 February, 2011, pp. 116-119.

[11] J. Campos Nobre, P. A. Pinheiro Rosa Duarte, L. Zambenedetti Granville, and L. M. Rockenbach Tarouco, "Delay-tolerant management using self-* properties and P2P technology," in IFIP/IEEE International Symposium on Integrated Network Management (IM 2013), Ghent, Belgium, 27-31, pp. 728-731, May 2013.

[12] A. Krifa, C. Barakat, and T. Spyropoulos, "Optimal Buffer Management Policies for Delay Tolerant Networks," in 5th
Annual IEEE Communications Society Conference on Sensor, Mesh and Ad Hoc Communications and Networks (SECON '08) San Francisco, CA, USA, 16-20 June, 2008, pp. 260-268.

[13] Y. Hsu and C. Hu, "Enhanced Buffer Management for Data Delivery to Multiple Destinations in Delay Tolerant Networks," IEEE Transactions on Vehicular Technology, vol. PP, Issue 99, pp. 1-1, 2015.

[14] K. Shin and S. Kim, "Enhanced buffer management policy that utilises message properties for delay-tolerant networks," IET Communications, vol. 5, Issue 6, pp. 753-759, 2011.

[15] H. Kazemi, G. Hadjichristofi, and L. A. DaSilva, "MMAN - a monitor for mobile ad hoc networks: design, implementation, and experimental evaluation," presented at the Proceedings of the third ACM international workshop on Wireless network testbeds, experimental evaluation and characterization, San Francisco, California, USA, 2008.

[16] J. N. Isento, J. A. Dias, F. Canelo, J. J. P. C. Rodrigues, and M. L. Proenca, "Moni4VDTN: A monitoring system for vehicular delay-tolerant networks," in IEEE International Conference on Communications (ICC 2012), 10-15 June, 2012, pp. 1188-1192.

[17] B. F. Ferreira, J. N. Isento, J. A. Dias, J. J. P. C. Rodrigues, and Z. Liang, "An SNMP-based solution for vehicular delay-tolerant network management," in IEEE Global Communications Conference (GLOBECOM 2012), Anaheim, CA, USA, 3-7 December, 2012, pp. 250-255.

[18] V. N. G. J. Soares, F. Farahmand, and J. J. P. C. Rodrigues, "VDTNsim: A simulation tool for vehicular delay-tolerant networks," in 15th IEEE International Workshop on Computer Aided Modeling, Analysis and Design of Communication Links and Networks (CAMAD 2010), 3-4 Dec. 2010, 2010, pp. 101105.

[19] J. A. F. F. Dias, J. N. G. Isento, B. M. C. Silva, V. N. G. J. Soares, and J. J. P. C. Rodrigues, "Performance assessment of IP over vehicular delay-tolerant networks through the VDTN@Lab testbed," EURASIP Journal on Wireless Communications and Networking, vol. 2012, Issue 1, pp. 1-12, 2012. 